\title{
Characteristic Vectors in p/q-Channel Orthonormal Wavelet
}

\author{
Zhaofeng $\mathrm{Li}^{1,2}$, Hongying Xiao ${ }^{1,2, *}$ \\ ${ }^{1}$ College of Science, China Three Gorges University, Yichang, China \\ ${ }^{2}$ Three Gorges Mathematical Research Center, China Three Gorges University, Yichang, China
}

\section{Email address:}

Kelly0128@163.com (Zhaofeng Li),30490652@qq.com(Hongying Xiao)

${ }^{*}$ Corresponding author

\section{To cite this article:}

Zhaofeng Li, Hongying Xiao. Characteristic Vectors in p/q-Channel Orthonormal Wavelet. Applied and Computational Mathematics. Vol. 8, No. 3, 2019, pp. 65-69. doi: 10.11648/j.acm.20190803.13

Received: July 8, 2019; Accepted: August 23, 2019; Published: August 27, 2019

\begin{abstract}
Wavelet analysis is a newly rapidly developing subject in the late twentieth century. As a time-frequency analysis tool, wavelet analysis has many advantages over other time-frequency tools, such as in signal processing, image processing, speech processing, pattern recognition, quantum physics and other fields. Multiresolution analysis (MRA for short) is an important method for studying wavelet orthonormal wavelet bases with rational dilation 2 . However, p/q-band wavelet is known to have advantages over 2-band wavelet in some aspects such as in signal processing and attracted more and more interest in recent years. But there are relatively less results for the case of p/q-band. This paper studies the orthonormal wavelet bases with rational dilation factor $\mathrm{p} / \mathrm{q}$ based on multiresolution analysis by a polyphase decomposition technique. First, we gave the concept of Multiresolution analysis with rational dilation $\mathrm{p} / \mathrm{q}$ and deduced an identity of the masks matrix. Also, a perfect reconstruction condition in terms of masks was presented. Further, we gave the refinement and wavelet matrices respectively and derived the characteristic roots and the corresponding orthonormal characteristic vectors of the wavelet matrix, and then a method with characteristic vectors was reduced to achieve the orthonormal wavelet bases with rational dilation factor p/q. In the end, an example is offered to verify this theory.
\end{abstract}

Keywords: Orthonormal Wavelet Base, Rational Dilation Factor, Perfect Reconstruction Condition

\section{Introduction}

The concept of wavelet transform was first proposed in 1974 by J. Morlet who was an French engineer engaged in oil signal processing. The study of wavelet bases and frames is the foundation of wavelet theory. For a long time, the construction of wavelet bases or frames was difficult until 1986 that Mallat and Meyer [1, 2] formulated the framework of the multiresolution analysis. This method makes the construction of wavelet base systematic. However, up to now, most studied cases are for integer dilation factors 2 . In fact in multi-rate digital processing, decimation by an $M>2$ ratio has superiorities over $M=2$ and some results have been applied to the scope of engineering [3-5, 8-12]. When we need to decomposition a signal, such as music, into sharper bands, maybe the non-integers are more suitable. In 1989, Auscher [4] has proved that wavelet bases with non-integer dilation factors are also possible. Recently, there are more and more studies in this fields. For example, Iiker Bayram and Tvan W. Selesnick [5] studied the orthonormal and overcomplete wavelet transforms based on rational sampling factors and presented several specific examples of Daubechies-type filters for a discrete orthonormal rational wavelet transform. Marcin Bownik and Darrin Speegle [6] discussed the wavelet dimension function for real dilations. In this paper, we study the orthonormal wavelet bases in terms of the refinement and wavelet masks, show the similar perfect reconstruction condition as the case of dilation factor 2 and deduce a characteristic vectors method to achieve an orthonormal wavelet base. With the development of wavelet transform, it is used in more and more fields, such as signal analysis, image processing, quantum mechanics and theoretical physics, intelligence of military electronic warfare and weapons, computer classification and recognition, artificial synthesis of music and language etc. We start by giving some concepts associated with multiresolution analysis (MRA for short).

\section{Notations and Preliminaries}

In this section, we will give some notations and 
preliminaries which will be used in the following sections. Let us first introduce the notion of MRA associated with a dilation factor $M \in R, M \geq 1$. Fix an integer $M$, an MRA is a family of nested subspaces $\left\{V_{j}\right\}_{j \in Z}$ of $L^{2}(R)$ such that

1. $\left\{V_{j}\right\} \subset\left\{V_{j+1}\right\}, \quad j \in Z$;

2. $\bigcap_{j \in Z} V_{j}=\{0\}, \overline{\bigcup_{j \in Z} V_{j}}=L^{2}(R)$;

3. $f(x) \in V_{j}$ if and only if $f(M x) \in V_{j+1}$;

4. There exists a function $\phi(x) \in V_{0}$ such that $\{\phi(x-n): n \in Z\}$ forms an orthonormal basis in $V_{0}$.

The function $\phi(x)$ is called a scaling function for the MRA. We emphasize that $\phi(x)$ in this paper is assumed to be compactly supported. When $M=2$, this is the classical definition of an MRA. MRA is an important method to construct and character the wavelets orthonormal bases and there are many results in this case [1-3]. If replace "an orthonormal basis" in the above definition by "a frame", we obtain the concept of frame multiresolution analysis (FMRA for short) which is another important method to study wavelet. In the other hand, the dilation factor $\mathrm{M}>2$ also is worth studying and there are academic achievements in this fields. For examples, Sun et al studied the construction of 3-band wavelet frames with symmetric properties by parameterizations of masks [7]. In 2007, Huang and Cheng presented a sufficient condition for an FMRA to form a tight wavelet frame and give an explicit constructing formula of wavelet tight frames by characteristic vectors [14]. As noted previously, the case of noninteger has advantages over 2-band wavelet orthonormal bases in some aspects such as in signal processing, intelligence of military electronic warfare and weapons, etc. In recent years, $\mathrm{P} / \mathrm{q}$ wavelet base attracted more and more interest and a few relevant literatures appeared in succession [8-12]. One may well wonder what the rational is for these fractional dilation factors. One of the answers is that they may provide a sharper frequency localization. For some applications, it may be useful to have wavelet bases that have a bandwidth narrower than one octave, and fractional dilation wavelet bases are one possible answer [3]. In this paper, we discuss the case $M=p / q,(p$ and $q$ are relatively prime integers with $p>q>1)$, namely $\operatorname{MRA}\left(\frac{p}{q}\right)$.

Let $\Psi=\left\{\psi^{1}, \psi^{2}, \cdots \psi^{N}\right\} \subset V_{1}$ and the generated family of $\Psi$ is

$$
\left\{\psi_{j, k}^{l}(x)=\left(\frac{p}{q}\right)^{\frac{j}{2}} \psi^{l}\left(\left(\frac{p}{q}\right)^{j} x-k q\right) ; j, k \in Z, l=1,2 \cdots N\right\} .
$$

When (1) forms an orthonormal base in $L^{2}(R)$, we say $\Psi$ is an $\operatorname{MRA}\left(\frac{p}{q}\right)$ wavelet associated with the scaling functions $\phi(x)$. Auscher [4] proved that when $M=p / q, p$ and $q$ are relatively prime integers with $p>q>1$, there exists a set of $p-q$ wavelet functions $\psi^{1}, \psi^{2}, \cdots \psi^{p-q}$ in $W_{0}=V_{1}-V_{0}$ such that (1) forms an orthonormal wavelet base for $L^{2}(R)$. It is well known that when $M=2$, the wavelet function is completely determinated by their masks [2]. What about the case $M=p / q$ ? Fortunately, the answer is yes. Since the structure of $\operatorname{MRA}\left(\frac{p}{q}\right)$ is very different from that of $\operatorname{MRA}(2)$, we need to introduce it briefly as follow.

From the above definition of $\operatorname{MRA}\left(\frac{p}{q}\right)$, we know that $V_{0} \subset V_{1}=\overline{\operatorname{Span}\left\{\phi\left(\frac{p}{q} x-n\right), n \in Z\right\}}$. Note $V_{0}$ is not a shift-invariant space and $W_{0}=V_{1}-V_{0}$ is shift-invariant under the translations by $k q, k \in Z$, hence

$$
\left\{\begin{array}{c}
\phi(x-m)=\sum_{n \in Z} h_{n}^{m} \phi\left(\frac{p}{q} x-n\right) \\
\psi^{l}(x)=\sum_{n \in Z} g_{n}^{m} \phi\left(\frac{p}{q} x-n\right)
\end{array}\right.
$$

here and through, $m=0,1, \cdots, q-1$ and $l=0,1, \cdots, p-q$.

The Fourier transforms of (2) are

$$
\begin{gathered}
\left\{\begin{array}{l}
\hat{\phi}(\omega) e^{-i \omega m}=\frac{q}{p} \sum_{n \in Z} h_{n}^{m} e^{-i \frac{q}{p} \omega n} \hat{\phi}\left(\frac{q}{p} \omega\right) \\
\hat{\psi}^{l}(\omega)=\frac{q}{p} \sum_{n \in Z} g_{n}^{m} e^{-i \frac{q}{p} \omega n} \hat{\phi}\left(\frac{q}{p} \omega\right) \\
\operatorname{Set}_{m}(\omega)=\frac{q}{p} \sum_{n \in Z} h_{n}^{m} e^{-i \omega n} \text { and } G^{l}(\omega)=\frac{q}{p} \sum_{n \in Z} g_{n}^{l} e^{-i \omega n}, \text { then }
\end{array}\right. \\
\left\{\begin{array}{l}
\hat{\phi}(\omega)=H_{m}\left(\frac{q}{p} \omega\right) \hat{\phi}\left(\frac{q}{p} \omega\right) e^{i \omega n} \\
\hat{\psi}^{l}(\omega)=G^{l}\left(\frac{q}{p} \omega\right) \hat{\phi}\left(\frac{q}{p} \omega\right)
\end{array}\right.
\end{gathered}
$$

The $2 \pi$ periodic functions $H_{m}(\omega)$ and $G^{l}(\omega)$ are called the refinement and the wavelet masks respectively. Let these masks forms a matrix $M(\omega)$ as

$$
M(\omega)=\left[\begin{array}{cccc}
H_{0}(\omega) & H_{0}\left(\omega+\frac{2 \pi q}{p}\right) & \cdots & H_{0}\left(\omega+\frac{2 \pi q(p-1)}{p}\right) \\
\vdots & \vdots & \vdots & \vdots \\
H_{q-1}(\omega) & H_{q-1}\left(\omega+\frac{2 \pi q}{p}\right) & \cdots & H_{q-1}\left(\omega+\frac{2 \pi q(p-1)}{p}\right) \\
G^{1}(\omega) & G^{1}\left(\omega+\frac{2 \pi q}{p}\right) & \cdots & G^{1}\left(\omega \frac{2 \pi q(p-1)}{p}\right) \\
\vdots & \vdots & \cdots & \vdots \\
G^{p-q}(\omega) & G^{p-q}\left(\omega+\frac{2 \pi q}{p}\right) & \cdots & G^{p-q}\left(\omega+\frac{2 \pi q(p-1)}{p}\right)
\end{array}\right] .
$$

Daubechies [3] discussed the case $M=\frac{3}{2}$ and concluded when the matrix $M(\omega)$ is unitary, $\phi(x)$ was an orthonormal wavelet. Actually, if $M(\omega)$ satisfies

$$
M(\omega) M^{*}(\omega)=q I_{p \times p}, \quad \text { a.e. } \omega .
$$


Here $M^{*}(\omega)$ represents the complex conjugate of the transpose of $M(\omega)$, and we can obtain an orthonomal wavelet via MRA. This is very similar the perfect reconstruction condition in the case of $M=2$ [2]:

Lemma (The perfect reconstruction condition) Let $\phi(x) \in L^{2}(R)$ with $\hat{\phi} \in L^{\infty}, \hat{\phi}$ continuous at 0 , and $\hat{\phi}(0)=1$ be a frame refinable function. $M(\omega)$ is the masks matrix of $\phi(x)$ :

$$
M(\omega)=\left[\begin{array}{ll}
H_{0}(\omega) & H_{0}(\omega+\pi) \\
H_{1}(\omega) & H_{1}(\omega+\pi)
\end{array}\right]
$$

then $M(\omega)$ satisfies:

$$
M(\omega) M^{*}(\omega)=I, \text { a.e. } \omega .
$$

The wavelet transform is $t$ tool that cuts up data or functions or operators into different frequency components and then studies each component with a resolution matched to its scale [3]. With the develop of MRA, a natural framework for the understanding and construction of new smooth orthonormal bases, the orthonormal wavelet bases and even wavelet frames became more and more interesting and constructed. Based on MRA, we can decomposed a signal or a function, etc. To different frequency components and then by the above perfect reconstruction condition, we then can obtain this signal. Thus, we can analysis a signal to achieve that condens a signal or remove noise from a signal.

\section{The Main Conclusions}

In this section, we deduced how an orthonormal wavelet base with dilation $\mathrm{p} / \mathrm{q}$ was obtained if the refinement masks were given. We call this method the characteristic vectors method and one of the advantages is that this method is easily implemented in the computer. This method was inspired by the results of $[3,13]$ and is based on the condition (5), so we analyze (5) first. Note that the columns of (5) are correlative makes it difficult to obtain an unitary matrix $M(\omega)$. We should eliminate this feature. To do so, we introduce a polyphase decomposition technique [7]. It is worth to illustrate that the case [7] discussed is integer $M \geq 3$, while here the case concerns non-integer $p / q$. The polyphase decomposition is decompose a $2 \pi$-periodic function into $p$ $\frac{2 \pi}{p}$-periodic functions. For example, we decompose $H_{0}(\omega)$ as

$$
H_{0}(\omega)=\sum_{k=0}^{p-1} e^{-i k \omega} H_{0, k}(\omega)
$$

Then

$$
\begin{aligned}
& H_{0}\left(\omega+\frac{2 \pi q L}{p}\right) \\
= & \sum_{k=0}^{p-1} e^{-i k\left(\omega+\frac{2 \pi q L}{p}\right)} H_{0, k}(\omega+2 \pi q L)=\sum_{k=0}^{p-1} e^{-i k\left(\omega+\frac{2 \pi q L}{p}\right)} H_{0, k}(\omega),
\end{aligned}
$$

thus $M(\omega)$ is equivalent to $M(\omega)=E(\omega) N(\omega)$

Where

$$
E(\omega)=\left[\begin{array}{cccc}
1 & e^{-i \omega} & \cdots & e^{-i \omega(p-1)} \\
1 & e^{-i \omega} e^{-i \frac{2 \pi q}{p}} & \cdots & e^{-i \omega(p-1)} e^{-i \frac{2 \pi q(p-1)}{p}} \\
\vdots & \vdots & \vdots & \vdots \\
1 & e^{-i \omega} e^{-i \frac{2 \pi q(p-1)}{p}} & \cdots & e^{-i \omega(p-1)} e^{-i \frac{2 \pi q(p-1)^{2}}{p}}
\end{array}\right]
$$

And

$$
N^{T}(\omega)=\left[\begin{array}{cccc}
H_{00}(\omega) & H_{01}(\omega) & \cdots & H_{0, p-1}(\omega) \\
H_{10}(\omega) & H_{11}(\omega) & \cdots & H_{1, p-1}(\omega) \\
\vdots & \vdots & \vdots & \vdots \\
H_{q-1,0}(\omega) & H_{q-1,1}(\omega) & \cdots & H_{q-1, p-1}(\omega) \\
G^{10}(\omega) & G^{11}(\omega) & \cdots & G^{1, p-1}(\omega) \\
\vdots & \vdots & \vdots & \vdots \\
G^{p-q, 0}(\omega) & G^{p-q, 1}(\omega) & \cdots & G^{p-q, p-1}(\omega)
\end{array}\right],
$$

$N^{T}(\omega)$ is the transpose of $N(\omega)$.

Equals

$$
N^{*} N=\frac{q}{p} I_{p \times p} \text {, a.e. } \omega .
$$

looks very like (5), but the rows of $N(\omega)$ are discorrelative. For the convenience of our discuss, redefine $N(\omega)$ as $\sqrt{\frac{q}{p}} N(\omega)$, then $N^{*} N=I_{p \times p}$. Define the refinement and wavelet matrices respectively

$$
R(\omega)=\left[\begin{array}{cccc}
H_{00}(\omega) & H_{10}(\omega) & \cdots & H_{q-1,0}(\omega) \\
H_{01}(\omega) & H_{11}(\omega) & \cdots & H_{q-1,1}(\omega) \\
\vdots & \vdots & \vdots & \vdots \\
H_{0, p-1}(\omega) & H_{1, p-1}(\omega) & \cdots & H_{q-1, p-1}(\omega)
\end{array}\right]
$$

and

$$
\mathrm{W}(\omega)=\left[\begin{array}{cccc}
G^{10}(\omega) & G^{20}(\omega) & \cdots & G^{\mathrm{p}-\mathrm{q}, 0}(\omega) \\
G^{11}(\omega) & G^{21}(\omega) & \cdots & G^{\mathrm{p}-\mathrm{q}, 1}(\omega) \\
\vdots & \vdots & \vdots & \vdots \\
G^{1, \mathrm{p}-1}(\omega) & G^{2, \mathrm{p}-1}(\omega) & \cdots & G^{\mathrm{p}-\mathrm{q}, \mathrm{p}-1}(\omega)
\end{array}\right] .
$$

Then we have $N(\omega)=[R(\omega), W(\omega)]$ and

$$
W(\omega) W^{*}(\omega)=I_{p \times p}-R(\omega) R^{*}(\omega) .
$$


The right of (7) is calculated and the left is a complex symmetric matrix. If the characteristic roots of $W(\omega) W^{*}(\omega)$ are $\lambda_{1}, \lambda_{2} \cdots \lambda_{p}$ and the corresponding orthonormal characteristic vectors are $\alpha_{1}, \alpha_{2} \cdots \alpha_{p}$ by count, then

$$
\begin{aligned}
& W(\omega) W^{*}(\omega) \\
= & \left(\alpha_{1}, \cdots \alpha_{p}\right) \operatorname{diag}\left(\lambda_{1}, \cdots \lambda_{p}\right)\left(\alpha_{1}, \cdots \alpha_{p}\right)^{*} \\
= & \left(\alpha_{1}, \cdots \alpha_{p}\right) \operatorname{diag}\left(\sqrt{\lambda_{1}}, \cdots \sqrt{\lambda_{p}}\right) \operatorname{diag}\left(\sqrt{\lambda_{1}}, \cdots \sqrt{\lambda_{p}}\right)^{*}\left(\alpha_{1}, \cdots \alpha_{p}\right)^{*} \\
= & \left(\alpha_{1}, \cdots \alpha_{p}\right) \operatorname{diag}\left(\sqrt{\lambda_{1}}, \cdots \sqrt{\lambda_{p}}\right) G(\omega)\left(\left(\alpha_{1}, \cdots \alpha_{p}\right) \operatorname{diag}\left(\sqrt{\lambda_{1}}, \cdots \sqrt{\lambda_{p}}\right) G(\omega)\right)^{*}
\end{aligned}
$$

here $G(\omega) G(\omega)^{*}=I_{p \times p}$.

This means, we can achieve $W(\omega)$ from the characteristic roots and vectors. In order to demonstrate it, we provide an example in the next section.

\section{Example}

In this section, we give one example in the case of $M=\frac{3}{2}$ to show explicitly how this method works. When $M=\frac{3}{2}$, the corresponding matrixes $N(\omega)$ and $W(\omega)$ are

$$
\begin{gathered}
N(\omega)=\left[\begin{array}{lll}
H_{00}(\omega) & H_{10}(\omega) & G^{10}(\omega) \\
H_{02}(\omega) & H_{11}(\omega) & G^{11}(\omega) \\
H_{02}(\omega) & H_{12}(\omega) & G^{12}(\omega)
\end{array}\right] \text { and } W(\omega)=\left[\begin{array}{l}
G^{10}(\omega) \\
G^{11}(\omega) \\
G^{12}(\omega)
\end{array}\right] . \\
W(\omega) W^{*}(\omega) \text { is } \\
{\left[\begin{array}{ccc}
1-\left|H_{00}\right|^{2}-\left|H_{10}\right|^{2} & -H_{00} \overline{H_{01}}-H_{10} \overline{H_{11}} & -H_{00} \overline{H_{02}}-H_{10} \overline{H_{12}} \\
-\overline{H_{00}} H_{01}-\overline{H_{10}} H_{11} & 1-\left|H_{01}\right|^{2}-\left|H_{11}\right|^{2} & -H_{01} \overline{H_{02}}-H_{11} \overline{H_{12}} \\
-\overline{H_{00}} H_{02}-\overline{H_{10}} H_{12} & -\overline{H_{01}} H_{02}-\overline{H_{11}} H_{12} & 1-\left|H_{02}\right|^{2}-\left|H_{12}\right|^{2}
\end{array}\right]}
\end{gathered}
$$

The characteristic roots of $W(\omega) W^{*}(\omega)$ are $\lambda_{1}=\lambda_{2}=0, \lambda_{3}=1$ and the orthogonal characteristic vectors are

$$
\left\{\begin{array}{c}
\alpha_{1}=\left(0,-H_{00} \overline{H_{02}}-H_{10} \overline{H_{12}}, H_{00} \overline{H_{01}}+H_{10} \overline{H_{11}}\right)^{T} \\
\alpha_{2}=\frac{1}{\left\|\alpha_{1}\right\|}\left(\left(\left|H_{00}\right|^{2}+\left|H_{10}\right|^{2}\right)\left(H_{01} \overline{H_{02}}+H_{11} \overline{H_{12}}\right)\right. \\
\left(H_{00} \overline{H_{01}}+H_{10} \overline{H_{11}}\right)\left(H_{01} \overline{H_{02}}+H_{11} \overline{H_{12}}\right), \\
\left.\quad\left(\overline{H_{00}} H_{01}+\overline{H_{10}} H_{11}\right)\left(\left|H_{02}\right|^{2}+\left|H_{12}\right|^{2}-1\right)\right)^{T} \\
\alpha_{3}=\left(\overline{H_{11} H_{02}}-\overline{H_{01}} H_{12}, \overline{H_{00} H_{12}}-\overline{H_{10} H_{02}},\right. \\
\left.\overline{H_{10} H_{01}}-\overline{H_{00} H_{11}}\right)^{T}
\end{array}\right.
$$

then $W(\omega)=\left(\alpha_{1}, \alpha_{2}, \alpha_{3}\right) \operatorname{diag}(0,0,1) G(\omega)$. In this case, the decompositions of wavelet are $\alpha_{3} g(\omega)$, here $g(\omega)=1$.

Let $V_{j}$ be the collection of functions whose Fourier Transforms supported in $\left[-\pi\left(\frac{3}{2}\right)^{j}, \pi\left(\frac{3}{2}\right)^{j}\right]$ and $\phi(x)=\frac{\sin \pi x}{\pi x}$, then $\hat{\phi}(\omega)=\chi_{[-\pi, \pi]}(\omega)$ and Auscher [4] verified that $\left\{\left\{V_{j}\right\}_{j \in Z}, \phi(x)\right\}_{\text {was }}$ an $\operatorname{MRA}\left(\frac{3}{2}\right)$. By a calculation, the two refinement masks are

$$
\begin{aligned}
& H_{0}(\omega)=\left\{\begin{array}{c}
1, \quad \omega \in\left[-\frac{2}{3} \pi, \frac{2}{3} \pi\right] \\
0, \quad \omega \in\left[-\pi,-\frac{2}{3} \pi\right) \cup\left(\frac{2}{3} \pi, \pi\right]
\end{array},\right. \\
& H_{1}(\omega)=\left\{\begin{array}{cc}
e^{-\frac{3}{2} i \omega}, \quad \omega \in\left[-\frac{2}{3} \pi, \frac{2}{3} \pi\right] \\
0, \quad \omega \in\left[-\pi,-\frac{2}{3} \pi\right) \cup\left(\frac{2}{3} \pi, \pi\right]
\end{array} .\right.
\end{aligned}
$$

And the corresponding polyphase decompositions of $H_{0}(\omega)$ and $H_{1}(\omega)$ are $H_{00}(\omega)=\frac{2}{3}$, a.e. $\omega \in[-\pi, \pi]$,

$$
\begin{aligned}
& H_{01}(\omega)= \begin{cases}-\frac{1}{3} e^{i \omega}, & \omega \in\left(-\pi,-\frac{2}{3} \pi\right) \cup\left(\frac{2}{3} \pi, \pi\right) \\
\frac{1}{3} e^{i \omega} e^{\frac{\pi}{3} i}, & \omega \in\left(-\frac{2}{3} \pi, 0\right) \\
\frac{1}{3} e^{i \omega} e^{-\frac{\pi}{3} i}, & \omega \in\left(0, \frac{2}{3} \pi\right)\end{cases} \\
& H_{02}(\omega)= \begin{cases}-\frac{1}{3} e^{2 i \omega}, & \omega \in\left(-\pi,-\frac{2}{3} \pi\right) \bigcup\left(\frac{2}{3} \pi, \pi\right) \\
\frac{1}{3} e^{2 i \omega} e^{-\frac{\pi}{3} i}, & \omega \in\left(-\frac{2}{3} \pi, 0\right) \\
\frac{1}{3} e^{2 i \omega} e^{\frac{\pi}{3} i}, & \omega \in\left(0, \frac{2}{3} \pi\right)\end{cases} \\
& \text { and } H_{10}(\omega)=0, \omega \in(-\pi, \pi),
\end{aligned}
$$

$$
H_{11}(\omega)=\left\{\begin{array}{cc}
-\frac{\sqrt{3}}{3} i e^{-\frac{i}{2} \omega}, & \omega \in\left(-\pi,-\frac{2}{3} \pi\right) \\
\frac{\sqrt{3}}{3} e^{-\frac{i}{2} \omega} e^{-\frac{\pi}{6} i}, & \omega \in\left(-\frac{2}{3} \pi, 0\right) \\
\frac{\sqrt{3}}{3} e^{-\frac{i}{2} \omega} e^{-\frac{\pi}{6} i}, & \omega \in\left(0, \frac{2}{3} \pi\right) \\
\frac{\sqrt{3}}{3} i e^{-\frac{i}{2} \omega}, & \omega \in\left(\frac{2}{3} \pi, \pi\right)
\end{array},\right.
$$

$$
H_{12}(\omega)=\left\{\begin{array}{ll}
\frac{\sqrt{3}}{3} i e^{\frac{i}{2} \omega}, & \omega \in\left(-\pi,-\frac{2}{3} \pi\right) \\
\frac{\sqrt{3}}{3} e^{\frac{i}{2} \omega} e^{\frac{\pi}{6} i}, & \omega \in\left(-\frac{2}{3} \pi, 0\right) \\
\frac{\sqrt{3}}{3} e^{\frac{i}{2} \omega} e^{-\frac{\pi}{6} i}, & \omega \in\left(0, \frac{2}{3} \pi\right) \\
-\frac{\sqrt{3}}{3} i e^{\frac{i}{2} \omega}, & \omega \in\left(\frac{2}{3} \pi, \pi\right)
\end{array} .\right.
$$

Finally, we obtain the orthonormal wavelet is

$$
G^{1}(\omega)=\left\{\begin{array}{c}
0, \quad \omega \in\left[-\frac{2}{3} \pi, \frac{2}{3} \pi\right] \\
\sqrt{2} g(\omega), \quad \omega \in\left[-\pi,-\frac{2}{3} \pi\right) \cup\left(\frac{2}{3} \pi, \pi\right]
\end{array} .\right.
$$

From the example above, we can see that this method is very straight and can be extended to the more general case $\mathrm{p} / \mathrm{q}$. When we do so, one point is worth illustration. Thay is, after p-q functions perpendicular to $H_{m}(\omega), m=0,1,2, \cdots q-1$ are achieved, the GramSchmidt orthogonalization should be used to ensure these $\mathrm{p}-\mathrm{q}$ functions are orthogonal to each other. 


\section{References}

[1] S. Mallat, "Multiresolution approximations and wavelet orthonormal bases of $\mathrm{L}^{2}$ (R)," Transactions of the American mathematical society, vol. 315, pp. 69-87.

[2] Meyer, Y., "Principe d'incertitude bases hibertiennes et algebres d'oerateurs," Sem. Bourbaki., 662 (1986).

[3] I. Daubechies, "Ten lectures on wavelets," CBMF conference series in applied mathematics 61, SIAM, Philadelphia, 1992.

[4] P. Auscher, "Wavelet bases for $\mathrm{L}^{2}$ (R) with rational dilation factor Wavelets and Their Applications," Jones and Barlett, Boston, 439-451, 1992.

[5] Ilker Bayram, Ivan W. Selesnick, "Design of orthonormal and overcomplete wavelet transforms based on rational sampling factors," Proc. SPIE 6763, 67630H (2007).

[6] Marcin Bownik, Darrin Speegle, "The wavelet dimension function for real dilations and dilations admitting non-MSF wavelets," Approximation Theory X: Wavelets, Splines, and Applications, 63-85, Vanderbilt University Press, 2002.

[7] Sun Qiyu, Bi Ning and Huang Daren, "An introduction to multiband wavelets," Zhejiang university press, 2001.

[8] M. K. Mihcak, I. Kozintsev, K. Ramchandran and P. Moulin, "Low-complexity image denoising based on statistical modeling of wavelet coefficients," IEEE Signal Processing Letters, 6 (1999), 300-303.

[9] A. K. Soman, P. P. Vaidyanathan and T. Q. Nguyen, "Linear phase paraunitary filter banks: theory, factorizations, and applications," IEEE Trans. Signal Processing, 41 (1993), 3480-3496.

[10] L. Gan and K. K. Ma, "A simplified lattice factorization for linear-phase perfect reconstruction filter bank," IEEE Signal Processing Letters, 8 (2001), 207-209.

[11] Chao, Zhang, et al. "Optimal scale of crop classification using unmanned aerial vehicle remote sensing imagery based on wavelet packet transform." Transactions of the Chinese Society of Agricultural Engineering (2016).

[12] Shleymovich M. P., M. V. Medvedev, and S. A. Lyasheva. "Object detection in the images in industrial process control systems based on salient points of wavelet transform analysis." International Conference on Industrial Engineering, Applications and Manufacturing IEEE, (2017): 1-6.

[13] A. Ron and Z. Shen, "Affine systems in $\mathrm{L}^{2}\left(\mathrm{R}^{\mathrm{d}}\right)$ : the analysis of the analysis operator," Journal of functional analysis, 148 (1997), 408-447.

[14] Y. D. Huang and Z. X. Cheng, "Explicit construction of wavelet tight frames with dilation factor a," Acta Mathematica Scientia, 2007, 27A (1), 7-18. 